\title{
Fiber positioning in microlens-fiber coupled integral field unit
}

\author{
Sabyasachi Chattopadhyay, ${ }^{\text {a,b, } * \text { Matthew A. Bershady, }}{ }^{\text {a,b,c }}$ Marsha J. Wolf, ${ }^{\text {a }}$ \\ Michael P. Smith, ${ }^{a}$ and Andrew Hauser ${ }^{a}$ \\ ${ }^{a}$ University of Wisconsin, Department of Astronomy, Madison, Wisconsin, United States \\ ${ }^{\mathrm{b}}$ South African Astronomical Observatory, Cape Town, South Africa \\ ${ }^{c}$ University of Cape Town, Department of Astronomy, Rondebosch, South Africa
}

\begin{abstract}
A generic fiber positioning strategy and a fabrication path are presented for microlens-fiber-coupled integral field units (IFUs). It is assumed that microlens-produced microimages are carried to the spectrograph input through a step-index, multimode fiber, but our results apply to micropupil reimaging applications as well. Considered are the performance trades between the filling percentage of the fiber core with the microimage versus throughput and observing efficiency. A merit function is defined as the product of the transmission efficiency and the étendue loss. For a hexagonal packing of spatial elements, the merit function has been found to be maximized to $94 \%$ of an ideal fiber IFU merit value (which has zero transmission loss and does not increase the étendue) with a microlens-fiber alignment (centering) tolerance of $1-\mu \mathrm{m}$ RMS. The maximum acceptable relative tilt between the fiber and the microlens face has been analyzed through optical modeling and found to be 0.3 deg RMS for input $f$-ratio slower than $f / 3.5$, but it is much more relaxed for faster beams. From the acceptable tilt, we have deduced a minimum thickness of the fiber holder to be $3 \mathrm{~mm}$ for $5 \mu \mathrm{m}$ clearance in hole diameter relative to the fiber outer diameter. Several options of fabricating fiber holders have been compared to identify cost-effective solutions that deliver the desired fiber positioning accuracy. Femto-second laser-drilling methods from commercial vendors deliver holes arrayed on plates with a relative position accuracy of $\pm 1.5-\mu \mathrm{m}$ RMS, similar diameter accuracy, and with an aspect ratio of 1:10 (diameter:thickness). One commercial vendor combines femtosecond laser-drilling with photolithographic etching to produce plates with thickness of $5 \mathrm{~mm}$, but with similar $( \pm 1-\mu \mathrm{m}$ RMS $)$ positioning accuracy and conical entry ports. Both of these techniques are found to be moderately expensive. A purely photolithographic technique performed at Wisconsin Center for Advanced Microelectronics (a facility at the University of Wisconsin, Madison), in tandem with deep reactive ion etching, has been used to produce a repeatable recipe with $100 \%$ yield. Photolithography is more precise $(0.5-\mu \mathrm{m}$ RMS) in terms of hole positioning and similar diameter accuracy $(1-\mu \mathrm{m}$ RMS $)$ but the plate can only have a thickness of $250 \mu \mathrm{m}$. () The Authors. Published by SPIE under a Creative Commons Attribution 4.0 Unported License. Distribution or reproduction of this work in whole or in part requires full attribution of the original publication, including its DOI. [DOI: 10.1117/1.JATIS.6.2.025002]
\end{abstract}

Keywords: multiobject spectroscopy; integral field unit; microlens; optical fiber.

Paper 20011 received Feb. 3, 2020; accepted for publication Jun. 16, 2020; published online Jun. $27,2020$.

\section{Introduction}

The use of two-dimensional arrays of optical fiber as an integral field unit (IFU) in astronomy has dramatically increased the efficiency of observations for extended sources such as galaxies and galactic nebulae. Optical fibers provide unprecedented flexibility in terms of instrument development and simplified observation. Early instruments, such as DensePak, ${ }^{1}$ used clusters of bare fibers to spatially format the sky and rearrange them into a one-dimensional slit at the spectrograph input. However, even the densest packing of fibers ${ }^{2,3}$ leaves interstitial regions between the fiber cores unsampled, due to the fiber shape, clad, and buffer. These gaps lead to fiber arrays

*Address all correspondence to Sabyasachi Chattopadhyay, E-mail: sabyasachi@saao.ac.za 
with only $\sim 60 \%$ on-sky fill factor. This underfill leads to an incompleteness in sampling extended sources [and often under-sampling the point spread function (PSF)] and often requires some sort of mitigation. Noncircular fibers do not significantly alter the situation because the noncircular cores are embedded in circular clad and buffer. The application of buffer stripping and light fusing ${ }^{4}$ can somewhat improve the on-sky fill factor but at the cost of increased focalratio degradation (FRD; see below), which introduces efficiency losses. Successful mitigation strategies have often followed the following two paths.

Historically, missing coverage due to fiber gaps has been mitigated through the dithering of bare-fiber arrays on the sky. Dithering is a process through which the IFU is moved with a slight offset between exposures to cover the interstitial regions. It has long been used in several instruments, such as SparsePak, ${ }^{5}$ PPak, ${ }^{2}$ VIRUS-P and VIRUS-W, ${ }^{6,7}$ and MaNGA. ${ }^{3}$ Hexagonal closepacking of fibers naturally leads to a three-point dither pattern, which helps obtain integral spatial coverage and resolution on par with the fiber core dimension. Despite these positives, ${ }^{8}$ analysis of dithered exposure can be challenging when observing conditions that change significantly from exposure to exposure. Dithering also demands high telescope offset and guiding precision (better than $0.1 \mathrm{arcsec}$ ), without which the photometric and astrometric calibration can be problematic. Additionally, atmospheric differential refraction (both chromatic and field) can make dithering an inaccurate procedure to implement for wide-field applications over a large range of hour angles. ${ }^{8}$

An alternative and elegant solution to filling the fiber gaps is to use a microlens array (MLA) or multiple MLAs in front of a fiber array. A well-positioned microlens system will collect and feed the light into the fiber. Such an optical system produces microimages or micropupils (depending on the optical design) of a continuous focal plane and positions them in a sparse 2-D array mimicking the position of the fiber cores in an array. This enables contiguous sampling of the focal plane and PSF, eliminating the need or at least reducing the importance of dithering the IFU and its associated challenges while retaining the flexibility of fibers to feed spectrographs at locations remote from the telescope focal surface. This approach has been taken, e.g., for the GMOS IFU on the Gemini telescopes, ${ }^{9}$ FLAMES and VIMOS on VLT ${ }^{10,11}$ and more recently for MEGARA on the GTC. ${ }^{12}$ In principle, MLAs can provide nearly complete coverage of a target (99\% with hexagonal MLA and uncovered corners). In practice, the corners often have inferior optical properties and are best masked, but they still deliver $\sim 90 \%$ integral coverage. $^{13,14}$

Other advantageous features of MLA coupling concern swapping telescope near- and farfield illumination for scrambling. An optical fiber scrambles the spatial information radially and azimuthally and modulates both near-field and far-field illumination. For a typical astronomical spectrograph, both near-field and far-field illumination are of astronomical importance: The near-field is imaged onto the detector, whereas the far-field determines the illumination of the optics and hence the aberrations that also contribute to the near-field image pattern (most astronomical spectrographs utilize optical designs that are far from diffraction limited). Various studies ${ }^{15-18}$ have shown that circular fibers are better at scrambling the far-field than rectangular or octagonal fibers, whereas noncircular fiber is superior for scrambling the near-field. The transposition of near- to far-field using lenslets and fiber in series can be used to optimize scrambling both for high-stability systems as well as moving-pupil telescopes such as Hobby-Eberly Telescope and SALT.

However, MLA coupling to fibers must be implemented with care to avoid significant degradation of instrument performance. Étendue-defined as the product of an optical instrument's collecting area $(A)$ and acceptance solid angle $(\Omega)$, or grasp $(A \times \Omega)$, with the total system throughput $(\eta)$-is typically considered a measure of an optical system's figure of merit. ${ }^{19}$ In the context of fiber optics, which serve to couple two imaging systems that conserve the area-solid-angle product (e.g., a telescope and a spectrograph), increases in grasp introduced by the fiber at constant throughput either (a) diminish the étendue of the overall system by overfilling down-stream optics, making for a lossier system; or (b) reduce the spectral resolution by increasing the entrance aperture. Throughput losses introduced by the fiber are additional losses to the system étendue. All of these cases can be viewed as a loss of information, and hence an increase in optical entropy. A fiber optic's grasp is defined as the product of the illuminated cross-sectional fiber-core area and the related solid angle of the illuminating beam, while the 
total throughput is the product of surface losses and bulk transmission. In the context of MLA coupling to fibers, it is critical to consider how this coupling may lead to increasing the grasp or reducing the throughput delivered by the fiber.

For example, MLAs offer an advantage of allowing for the fiber input $f$-ratio to be modulated in order to minimize FRD but have the potential disadvantage that misalignment between MLAs and fiber cores can lead to light loss and geometric FRD (gFRD). FRD is a phenomenon related to fibers, in which the input beam $f$-ratio becomes faster when exiting the fiber. There are several explanations of FRD that include, but are not limited to, fiber-polishing imperfections, ${ }^{20}$ end stress, ${ }^{21}$ and microbends. ${ }^{22}$ It is well understood that FRD cannot be entirely eliminated but can only be minimized through certain procedures. FRD may also introduce lossy modes leading to lower transmission. gFRD is introduced due to the misalignment of the input beam with the fiber optical axis and thus geometric in nature $;^{23}$ gFRD can be eliminated with proper optical design and alignment. Light-loss due to misalignment can be mitigated by underfilling the fiber cores with the MLA microimages, but this comes at a cost in entropy: fibers produce radial scrambling in the near-field, which, in the case of an under-filled fiber entrance aperture, tends to lead to a more filled fiber exit aperture (this radial scrambling is a function of fiber properties, including length, e.g., Fig. 8 in Ref. 24). This last point is the crux of the challenge with MLA-fiber coupling.

Ren and Allington-Smith ${ }^{19}$ explored in detail several of these key issues of fiber-microlens coupling. For example, they consider the important performance implications of under or overfilling the fiber input surface, including the effects of spherical aberration and diffraction from the microlens. In general, they conclude that the use of microlenses requires an oversize factor between the fiber core and microlens clear aperture, an equivalent of the mechanical aperture. This oversize factor impacts the effective slit width seen by the spectrograph (pseudoslit), and hence lowers spectral resolution at constant collimator focal-length and grating dispersion. However, their analysis is based on the use of plano-convex microlenses at the input and output of the fiber. This optical configuration is mechanically simple to implement and serves to transfer a micropupil image to and from the input and output fiber faces, respectively. As they note, this transfer implementation introduces nontelecentricity to the micropupil image, and hence introduces gFRD due to the azimuthal scrambling properties of the fibers even in the case of perfect fiber-microlens alignment. Their solution to this form of gFRD places limits on the input beam speed to the microlens to low values that are not optimal for other design considerations. They also identify an optimum input $f$-ratio and fiber core size based on the microbend theory for FRD that has not been confirmed in practice. In our study, we extend their work to provide: (i) a more generic treatment of the impact of fiber alignment and fill factor with respect to an optically defined chief ray for both micropupil and microimage transfer system; (ii) an explicit expectation that if the fiber input near-field is under-filled, the output end will be filled with near uniformity (while in detail the output near-field illumination will depend on fiber length, input fill factor, and centricity of the illumination ${ }^{25}$ ); and (iii) practical limits on lenslet radius of curvature (RoC). The latter is critical since it places constraints on the input beam speed to the lenslets, which connects back to the issue of gFRD in the context of lenslets used as pupil reimagers.

In our framework, we assume MLAs must feed telecentric images (pupil or near-field) to fibers at fast $f$-ratios $(\sim 3)$ to minimize the effect of FRD. On the other hand, the microlenses need to be fed at a slow $f$-ratios to ensure that their RoC is manufacturable. This difference in beam speeds, and in turn angular area $(\Omega)$, leads to a difference in surface area between the MLA and fibers, with fibers requiring lesser area than MLA. Thus the fiber array must be sparsely populated yet precisely matching the position of the micropupil/image array produced by the MLA. Positioning fibers as shown in Fig. 1 lead to positioning tolerance generated from position inaccuracy as well as diameter inaccuracy. This tolerance will affect the overall throughput of the system.

Consequently, the success of using MLAs in front of a fiber array in an IFU depends not only on the relative positioning of fibers and microlenses but also on the amount of the fiber core that is filled as well as minimization of tip/tilt error between the fiber and the microlens surface. In this paper, we will define merit functions that quantify this "success," and discuss the strategy to optimize the IFU design in Sec. 2. Section 3 describes one of the methods to implement the required fiber positioning. In Sec. 4, we summarize our findings. 


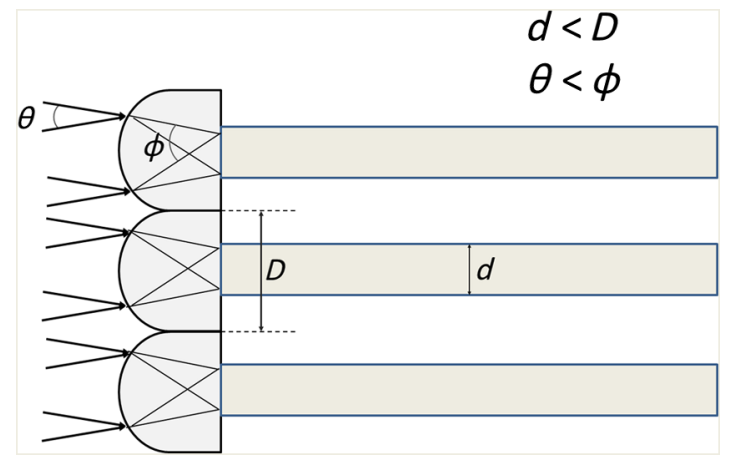

Fig. 1 The requirement of a sparsed fiber array for a microlens-fiber coupled IFU. The fiber diameter $(d)$ is smaller than microlens $(D)$ as the microlens requires a slower beam $(\theta)$ to be fed to be manufacturable, while the fiber needs a faster beam $(\phi)$ to minimize the FRD. Since this figure is presented to demonstrate the requirement of sparse distribution of fiber for a microlens feed IFU, note that the nontelecentric beam entering the fiber as shown in this figure would increase effective $\phi$ and in turn will increase FRD from its nominal value.

\section{Microlens Coupled Fiber IFU Design Strategy}

The key to developing a fiber-MLA coupled IFU is to optimize the trade-offs between throughput loss and increase in grasp such that optical entropy increase is minimized. For the purposes of this development, we will consider a system, in which the FRD and transmission properties due to the fiber alone to be fixed. We first isolate the trade-offs between the fraction of the fiber optical core that is filled by the MLA microimage and MLA-fiber centering precision. In Sec. 2.3, we consider the impact of gFRD on the requirements for fiber angular alignment.

Ideally, to achieve minimum entropy gain on the one hand it is desirable to fill the entire fiber entrance core since radial scrambling will tend to fill the exit core regardless of the entrance fill factor. However, in this fully filled input core scenario, the positioning accuracy of the microimage onto the fibers (or, alternatively, the fiber location) must be extremely high to minimize throughput loss at the fiber-microlens junction. Such high-precision position requirements can dominate the cost of fiber-based IFU development.

The minimum entropy gain also depends on the encircled energy (EE) within the fiber core for a given microimage diameter. If we define EE99 as the radius of $99 \%$ EE, clearly the quality of the microlens design should deliver a microimage that is at least as small as the fiber core radius.

Given these two considerations, it is important to determine the fraction of the fiber core to be filled by the microimage given the achievable alignment precision between fiber and MLA. Hence, a merit function is defined to find the exact fiber core fill fraction that includes a decenter model of the fiber array holder and EE distribution within the microimage. We define the fractional change in grasp due to the fiber coupling as

$$
E(A, \Omega)=\frac{A_{o} \Omega_{o}}{A_{i} \Omega_{i}},
$$

where $A_{i}$ and $A_{o}$ are input and output microimage areas, respectively, at the fiber face while $\Omega_{i}$ and $\Omega_{o}$ are the respective solid angles of the beams. For a microlens-fiber throughput of $\eta$, the merit function of a single spaxel design is then defined as

$$
M_{s}=\frac{\eta}{E(A, \Omega)}=\frac{\eta A_{i} \Omega_{i}}{A_{o} \Omega_{o}} .
$$

For a typical multimode fiber used in astronomy, $A_{o}$ remains fixed and equal to the fiber core size as the beam completely covers the fiber output face. Consequently, $M_{s}$ increases for an increase in input microimage size. $M_{s}$ also increases with increasing $\Omega_{i}$ since FRD modulates slower input beams more compared to the faster beams; whereas the ratio of $\Omega_{i} / \Omega_{o}$ remains less than unity due to FRD, it approaches unity as the input beam approaches the fiber numerical 
aperture (NA). (It is assumed in all of this discussion that the fiber input beam does not exceed the NA since this would be a lossy application.) Putting these factors together, we expect $M_{s}$ to be less than unity in practice. Our objective is to maximize $M_{s}$ by careful design of a microlens system that maximizes $\Omega_{i}$ for a suitable choice of $A_{i}$ within the achievable fiber positioning accuracy.

As long as the fiber positioning accuracy does not depend on the fiber spacing, $\Omega_{i}$ and $A_{i}$ can be decoupled so that we may consider the optimum $A_{i} / A_{o}$ ratio in the context of the positioning accuracy alone. For an appropriate statistical treatment let $F(p, \mu, \sigma)$ be a gaussian decenter distribution of fiber position $p$ with mean $\mu$ and standard deviation $\sigma$. The average of single spaxel merit function convolved with the decenter distribution is defined as the IFU merit function $M_{i}$ described as

$$
M_{i}=\left\langle M_{s} \circledast F(p, \mu, \sigma)\right\rangle=\left\langle\frac{\eta A_{i} \Omega_{i}}{A_{o} \Omega_{o}} \circledast F(p, \mu, \sigma)\right\rangle .
$$

Maximization of $M_{i}$ may be achieved through microlens-fiber optical design and ensuring relative positioning accuracy as described by Perez-Calpena et al. ${ }^{26}$ for MEGARA in GTC. The study treated the microlens positioning error within lenslet array and microlens to fiber positioning error as separate entity. Here $F(p, \mu, \sigma)$ is a combination of both of these errors. The metric has been used in Sec. 4.1 in finding the best fiber holder technique among the technologies available while defining the requirements for the optical design at the same time.

\subsection{Limit of Input Solid Angle}

Equation (3) suggests that to maximize the merit function, we must maximize the input solid angle $\Omega_{i}$ which would minimize the effects of FRD. Ideally, the NA of the fiber (typically 0.22) would put the upper limit on the $\Omega_{i}$ for bare fiber IFU. However, with microlens, the limit on $\Omega_{i}$ is defined by the requirement of lenslet clear aperture. We describe this through the figure shown in Fig. 2. The marginal ray of a collimated beam is going to pass through a plano-convex microlens and produce a micropupil/image at the back of the lenslet. We have considered a plano-convex lens just to visualize the scenario. Here $R, d, \theta_{i}, \theta_{r}, n$, and $f$ are the RoC, semidiameter of the clear aperture, input angle, refracted angle, refractive index, and lenslet focal length, respectively.

Since

$$
\sin \theta_{r}=\frac{d}{n R}
$$

we have

$$
\tan \theta_{r}=\frac{\sin \theta_{r}}{\cos \theta_{r}}=\frac{\frac{d}{n R}}{\sqrt{1-\left(\frac{d}{n R}\right)^{2}}}
$$

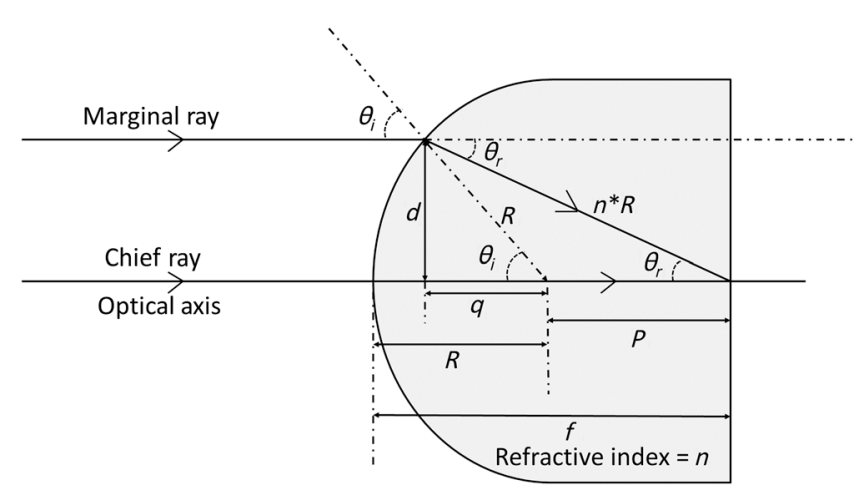

Fig. 2 Effect of aperture on the upper limit of the input solid angle. 
Also

$$
\tan \theta_{r}=\frac{d}{P+q} .
$$

Combining Eqs. (5) and (6), we find

$$
P=\sqrt{(n R)^{2}-d^{2}}-\sqrt{R^{2}-d^{2}} .
$$

The criteria for an acceptable clear aperture is: within the clear aperture, all the rays of a collimated beam parallel to the optical axis of the lenslet should pass through the focus. To ensure the refracted light must pass through the focus at the focal plane which is the backplane of the lenslet, this condition must be met

$$
P \leq f-R
$$

For a plano-convex lens using the lens-makers formula, we have

$$
R=(n-1) f
$$

Combining Eqs. (8) and (9):

$$
P \leq\left(\frac{2-n}{n-1}\right) R
$$

Finally, from Eqs. (7) and (10):

$$
\frac{2-n}{n-1} \geq \sqrt{n^{2}-\left(\frac{d}{R}\right)^{2}}-\sqrt{1-\left(\frac{d}{R}\right)^{2}}
$$

Equation (11) shows that, for the refractive index of fused silica, the value of $d$ can be as high as $0.65 \times R$. From Eq. (9), a limit on $R$ would put a limit on the focal length of the microlens. Again, for fused silica, $d$ is can only be as high as $29 \%$ of the focal length. This limit falls nicely along with the circular microlens profile, which is within the regime of a parabolic surface at $d=0.62 \times R$ and hence the spherical aberration is minimal. Even the remaining aberration is taken out by now-standard manufacturing processes that generate a refractive index profile decreasing from the lenslet center to the edge (Sec. 7.7 of Chapter 7 from Ref. 27). The manufacturing limit of the clear aperture is defined by the thickness of the substrate which primarily defines the shortest possible focal length by limiting the RoC. At the limit of $d \sim 0.65 \times R$, the NA (shag over semidiameter of clear aperture) gets limited to 0.22 , which also matches nicely with the NA of a typical multimode optical fiber. However, as described, the acceptable NA is dependent on the focal length and thus substrate thickness. Small substrate thickness $(\sim 100 \mu \mathrm{m})$ would demand a limiting NA of 0.14 only. For a given fiber core size and telescope $f$-ratio, theoretically, the microlens focal length and practically the substrate thickness acts as a link between the clear aperture and the maximum attainable $\Omega_{i}$ (Chattopadhyay et al. in preparation).

\subsection{Slit-Mask Integral Field Unit for SALT}

As an example of its generic description, we have implemented, analyzed, and discussed our theoretical merit function for the IFU optomechanical design of the slit-mask IFU (SMI), an instrument for the South African Large Telescope (SALT). As a pupil scrambling IFU, the current SMI design deploys 270 spaxels (spatially contiguous fiber-microlens elements) at the telescope science focal plane. The spaxels are hexagonal and each covers $\sim 1.33$ arc sec (corner to corner) on the sky. Physically, each hexagonal microlens is $\sim 370-\mu \mathrm{m}$ wide, transmits an $f / 4.2$ telescope beam as a part of the focal plane at the input of the fiber as a micropupil. 
SMI is expected to use $200-\mu \mathrm{m}$ core fibers. The fibers must be positioned at the back of microlens in a way that the fiber core engulfs the entire micropupil to avoid any throughput loss. However, it is also important to explore the pros and cons of overfilling the fiber core as well. It is possible to prepare the fiber array and reflect their positions on the MLA micropupil pattern. The custom positioning of microlenses in an MLA is significantly costlier than maneuvering the fiber positions. To ensure fiber positioning is accurate enough to minimize étendue loss, we break out the requirements into three components.

1. Fiber core centers must be aligned with microimage centers (positioning accuracy).

2. Hole diameters should be as close as possible to the fiber outer diameter (including clad and buffer; diameter accuracy).

3. Holes should be deep enough to limit tilt at the fiber face relative to microlens face to within the acceptable tolerance for nontelecentricity (hole depth).

Typically, fibers are plugged into holes in a plate, which we refer to as a fiber holder. The holder hole pattern, therefore, mimics the micropupil (pupil scrambling IFU) or microimage (image scrambling IFU) pattern.

\subsection{Angular (Tilt) Alignment Requirement}

The design and fabrication of a fiber holder depend on the requirements for sky-fiber positioning accuracy as well as spectrograph acceptable fiber output. The positioning accuracy is driven by the microimages produced by the MLA. However, the tip/tilt in the coupling face between a fiber and a microlens also defines the fiber output beam degradation. This kind of FRD is known as gFRD, which is introduced due to misalignment of the input beam with the fiber optical axis and thus geometric in nature. ${ }^{23}$ The acceptable limit of gFRD drives the tip/tilt tolerance between microlens and fiber.

We have performed a $\mathrm{Zemax}^{\odot}$ simulation to understand the effect of tip and tilt on the energy distribution at the fiber output for our example application of an SALT SMI. We have chosen the input beam speed to be $f / 4.2$, which is the telescope beam speed. The practical FRD measurement defines the expected EE98 for the purpose of simulation which is $f / 4$ in our case. In the simulation, a 1-m-long fiber of 300- $\mu \mathrm{m}$ core is fed with an $f / 4.2$ beam and the fiber output is captured on a surface at a distance of $5 \mathrm{~mm}$ from the fiber output face. At this surface,

\section{Encircled energy variation with output $f$-ratio for various input beam tilt at $f / 4.2$}

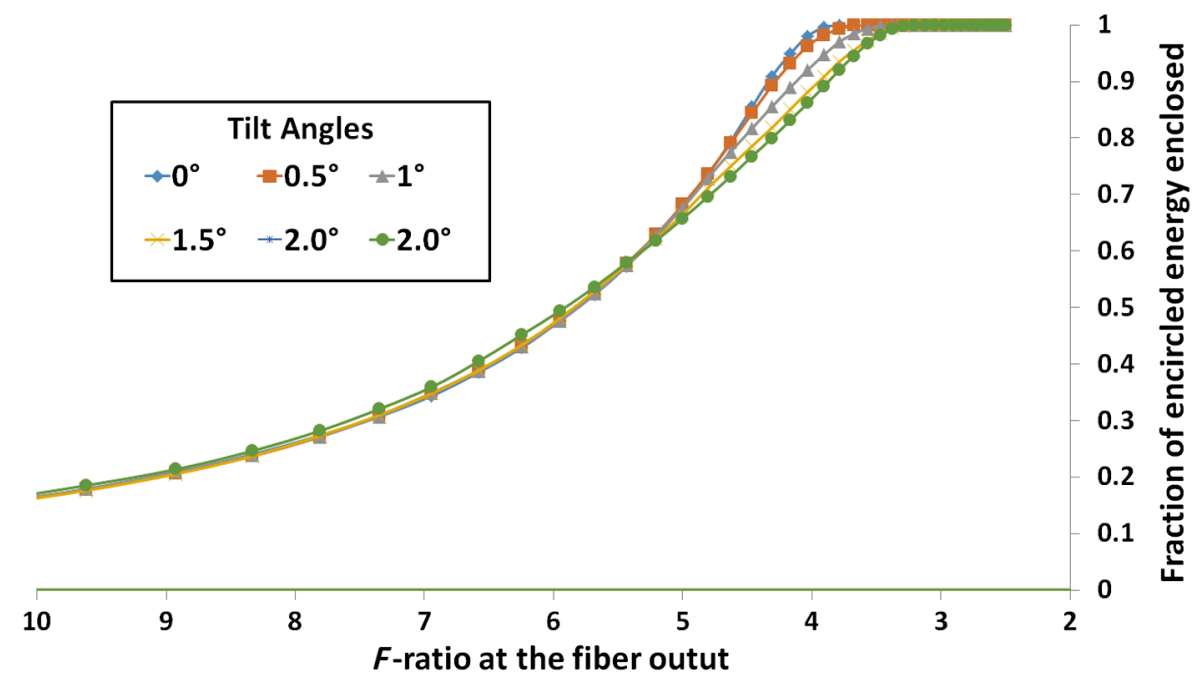

Fig. 3 EE variation with output $f$-ratio for a 1-m fiber fed with a $f / 4.2$ input beam and nontelecentric injection varying from 0 deg to $2 \mathrm{deg}$ at a step of $0.1 \mathrm{deg}$. Color of the curves different tilt angles in degree between fiber and microlens face. 


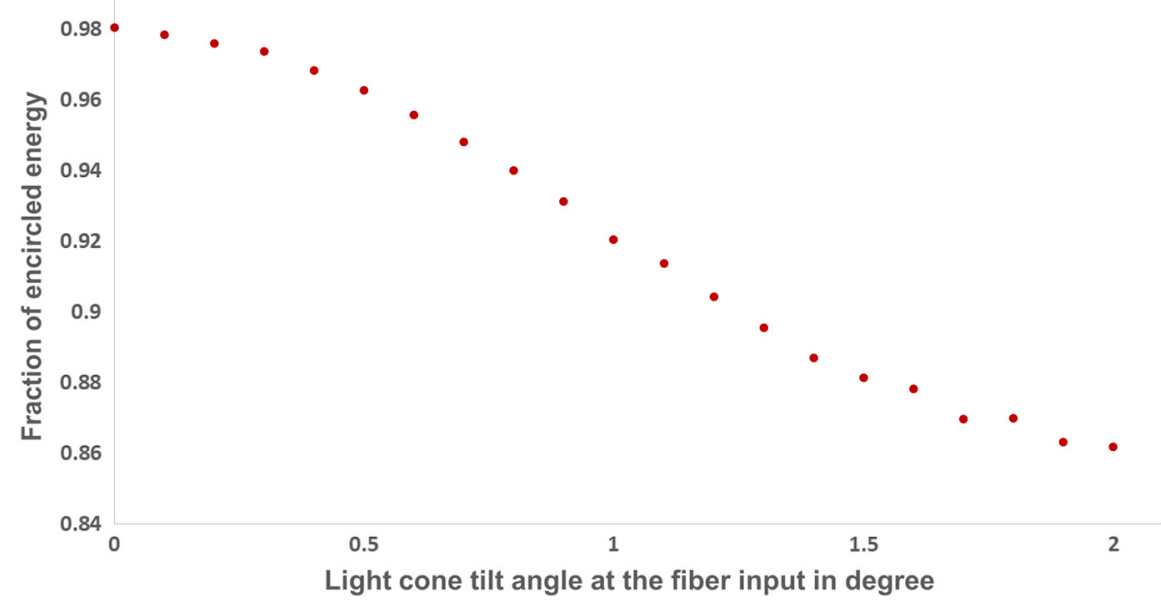

Fig. 4 Fiber output EE at $f / 4$ as a function of nontelecentric injection (tilt), as measure at a distance of $5 \mathrm{~mm}$ from fiber output face. Near-field effects due to the finite size of the fiber output face are $2 \%$ as reckoned from EE at zero tilt angle.

the extended source EE distribution is simulated using 5 million rays. The pupil of the optics does not coincide with the plane at $5 \mathrm{~mm}$. Although we have not calculated to what degree the finite size of the near-field image impacts the $\mathrm{EE}$ at $5 \mathrm{~mm}$, this is unimportant for our purposes, since all we care about is the degradation relative to no tilt, i.e., roughly $98 \% \mathrm{EE}$ as seen at the $y$ intercept. The input beam tilt (equivalent to the tilt between MLA and fiber) is varied from $0 \mathrm{deg}$ to $2 \mathrm{deg}$ at a step of $0.1 \mathrm{deg}$. The EE distribution is plotted against the output $f$-ratio in Fig. 3 for different tilt angles. From this distribution, an EE variation versus tilt is plotted for the output $f$-ratio of $f / 4$ in Fig. 4 . Until $0.3 \mathrm{deg}$, the EE does not vary significantly. However, there could be tilt introduced from other sources (e.g., MLA defects, global MLA misalignment, etc.) so we only allow a fraction $(33 \%=0.1 \mathrm{deg})$ of the total available budget to be attributed to the fiber angular misalignment. This acceptable tilt angle can be converted into the fiber-holder thickness depending on the difference between fiber diameter and fiber holder hole diameter. For example, to accept a 5- $\mu \mathrm{m}$ larger hole diameter than the fiber diameter (and hence 5- $\mu \mathrm{m}$ decenter error), the fiber holder thickness must be $\sim 3 \mathrm{~mm}$.

\section{Fiber Holder Development}

\subsection{Fiber Holder Development Techniques}

We have explored three technologies to develop fiber holders with the aim of determining the accuracy and precision achievable by these different techniques in relative hole position, diameter, and achievable hole depth. The three techniques are laser drilling, photolithography, and a combination of both.

- Sun-Light Tech. ${ }^{\odot}(S L T)$ uses a femtosecond-pulsed laser to drill metal or glass plates with an aspect ratio of 10:1 between hole depth and diameter. For a hole diameter of $280 \mu \mathrm{m}$ (5- $\mu \mathrm{m}$ larger than the fiber outer diameter including buffer for SMI), we can achieve a hole depth of $\sim 2.8 \mathrm{~mm}$. We have inspected an 1-mm thick $10 \times 10$ rectangular array of $100-\mu \mathrm{m}$ diameter holes spaced at $150-\mu \mathrm{m}$ center to center (refer to Fig. 5). The microscopic image was used to measure the hole position accuracy and hole diameter accuracy and it is found that both are better than 1.5- $\mu \mathrm{m}$ RMS.

- Wisconsin Center for Advanced Microelectronics (WCAM) currently Nanoscale Fabrication Center at the University of Wisconsin, Madison provides an ideal, in-house laboratory facility to perform photolithography. Photolithography is a chemical process that transfers patterns to a metal or glass plate. It has a much higher aspect ratio for hole 

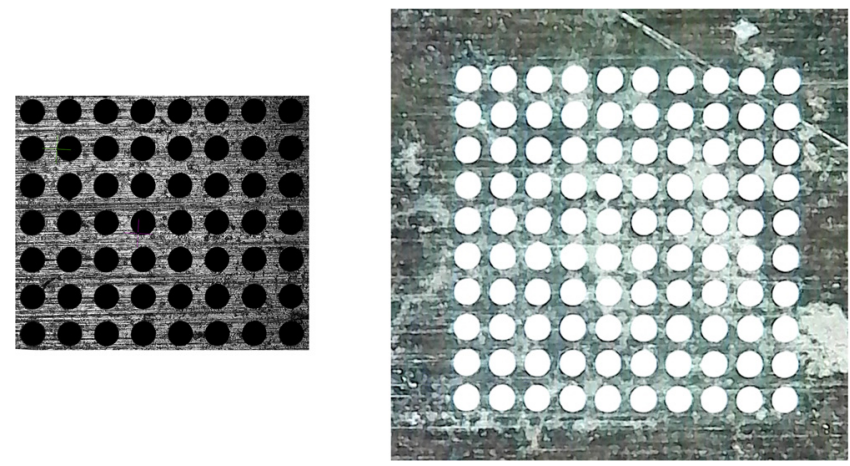

Fig. 5 Back and front illuminated image of SLT produced $10 \times 10$ hole array of $100 \mu \mathrm{m}$ holes with a pitch of $150 \mu \mathrm{m}$. The RMS positioning and diameter error was found to be $\pm 1.5 \mu \mathrm{m}$ measured using microscopic calibrated image.

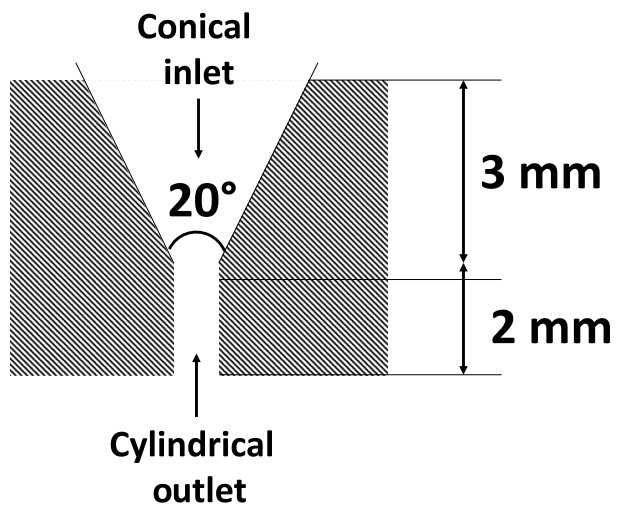

Fig. 6 FP design of hole as fiber holder that has a conical entry to the desired cylindrical hole which demands sparse fiber positioning.

depth to diameter (150:1) compared to laser-drilled technique, as well as higher accuracy in hole positioning $(\sim 0.5 \mu \mathrm{m})$ and diameter $(\sim 0.5 \mu \mathrm{m})$. We return to this technique in Sec. 3 .

- FemtoPrint ${ }^{\odot}(F P)$ is a Swiss manufacturer that uses a combination of the above two techniques to produce a fiber holder. A key feature of FP-produced holders is a conical hole at the entry surface which meets the expected cylindrical hole as shown in Fig. 6. FP-produced holders are expected to have 2- and 7- $\mu \mathrm{m}$ RMS hole diameter accuracy for 1- and 5-mm-thick holders, respectively. In both cases, the hole positioning accuracy for the cylindrical section is 5- $\mu \mathrm{m}$ RMS. Currently, FP is capable of delivering $\pm 1-\mu \mathrm{m}$ hole position and diameter accuracy over a holder thickness of $5 \mathrm{~mm}$. We term this capability/ distribution as modified FP.

\subsection{Lithography as a Tool for Pattern Transfer}

In this section, we describe the fabrication of a fiber holder through photolithography. We have used the facilities at WCAM for this purpose. Wet photolithography has previously been used ${ }^{28}$ to develop microlens-fiber coupled IFU for the Devasthal Optical Telescope Integral Field Spectrograph (DOTIFS). However, it has been shown that wet photolithography can have issues of irregular hole diameter, low etchable thickness (maximum of $100 \mu \mathrm{m}$ ), low throughput $(\sim 30 \%)$, etc. In dry photolithography, it is possible to use a much thicker substrate, such as a silicon wafer. We have used 250- and 500- $\mu \mathrm{m}$ dual-side polished (DSP) silicon wafers of $\langle 100\rangle$ crystal lattice orientation as our substrate. The DSP feature helped us maintain etching uniformity and hole isotropy while the $\langle 100\rangle$ orientation is found to be easier to etch with the existing apparatus than other orientations. 


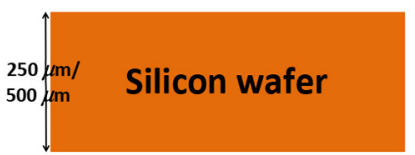

(a)

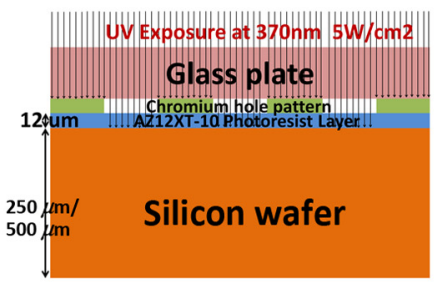

(d)

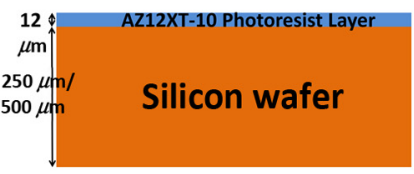

(b)

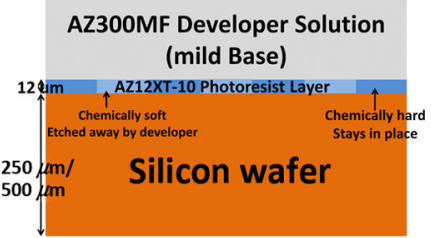

(e)

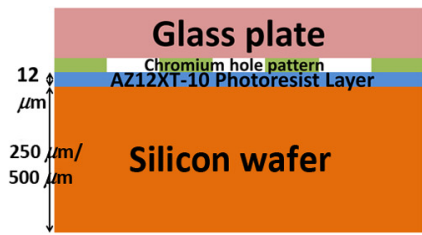

(c)

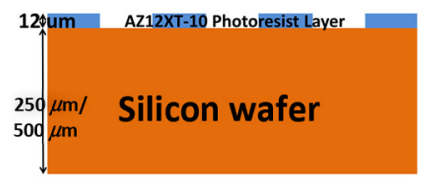

(f)

Fig. 7 Schematic of different steps involved in photolithographic procedure used toward developing a fiber holder at WCAM. (a) Clean silicon wafer, (b) deposition of photoresist layer, (c) alignment of chromium hole pattern, (d) UV exposure, (e) etching the photoresist with developer, and (f) wafer ready for etching process.

Photolithography is a chemical technique for precisely etching patterns on a silicon wafer. The positional accuracy of etching patterns can be controlled to a tenth of a micron using ultraviolet (UV) light of 365 to $370 \mathrm{~nm}$. For prototype development purposes, we have tried to fabricate a fiber holder that can hold one of the smaller size multimode fibers $(\sim 100-\mu \mathrm{m}$ core, $145 \mu \mathrm{m}$ including clad, jacket/buffer) usable for astronomical purpose. We believe if the method can hold position and diameter accuracy for smaller diameter and separation, it would be expected to perform the same for wider fibers with larger separation. Given our requirement for total hole depth and the limitations of silicon wafer thickness for etching, several wafers need to be fabricated and stacked to achieve a thickness of $3 \mathrm{~mm}$. This requirement has an impact on our etching design. Figure 7 illustrates the photolithographic process. We describe the process below with respect to this figure.

1. Photolithography requires the purest form of substrate. Impurities, if any, can act as a constructive or a destructive catalyst in the etching process leading to a higher or lower etching rate, and hence hole size and regularity within the stipulated etching time. Such impurities thus reduce the yield of acceptable arrays in a wafer. The wafer should also be easily polishable once bonded to the fibers. Since the fiber core and cladding are made of fused silica, silicon is used as the wafer substrate. The chosen silicon wafer has a low percentage $(<0.001 \%)$ of impurities. The glue, the wafer, and the fiber all have very similar thermal expansion properties and hence will not create any stress-induced internal mechanical issues (insofar as they are properly mounted) in observatory conditions where the temperature can vary from $-10 \mathrm{deg}$ to $25 \mathrm{deg}$.

2. The two factors that determine wafer dimensions are the number of masks that we want to produce at a time and the UV-illumination area. Six masks are fabricated in a single process which is sufficient for achieving a stack thickness of $3 \mathrm{~mm}$. (There can be added thickness of a few tens of $\mu \mathrm{m}$ by layers of glue between each pair of wafers.) The corner to corner dimension of a hexagonal IFU holder is $9.4 \mathrm{~mm}$. We have kept an additional 5.6- $\mathrm{mm}$ space on all four sides for ease of handling. On the other hand, the UV lamp assembly can illuminate an area corresponding to a circle of diameter $75 \mathrm{~mm}$. The wafer dimensions are large enough to create a $2 \times 3$ array of holders.

3. The success of photolithography depends on the choice of photoresist that gets applied to the wafer. Photoresist is a substance, that based on its state, may or may not pass photons of a specific wavelength through it. The first step of the process is to transfer the intended pattern to photoresist and use it as a mask to etch the wafer. We have used a positive photoresist solution AZ-12XT-10PR at a spin rate of $1500 \mathrm{rpm}$ (this is the lowest possible spin rate to get even thickness) that would help us to achieve a thickness of $12 \mu \mathrm{m}$. 
The requirement of photoresist thickness is defined by the etching time. For an etch time of $\sim 2.5 \mathrm{~h}$, the photoresist is expected to get 8 - to $10-\mu \mathrm{m}$ deep holes.

4. A lump of photoresist is applied on the wafer and the wafer was rotated for $30 \mathrm{~s}$. However, at this point, the photoresist is liquid and hence mechanically not stable to hold its position. Once a uniform layer has formed, the photoresist solution starts to harden due to the evaporation of the solvent thinner. This process is expedited by means of pre-exposure baking at $110^{\circ} \mathrm{C}$ for $180 \mathrm{~s}$.

5. For the next step, the desired pattern is created on a transparent glass slide. The pattern consists of circular transparent spots on chromium-coated opaque glass, which mimic the position and diameter of holes. Ideally, the spot centroid pattern of an MLA should be used to determine the location of the transparent patches for the corresponding mask. For general considerations, here we have used regularly spaced circles. The chromium-plated area is opaque to the UV light. The remaining part of the wafer does not hinder the transmission of the UV light.

6. The wafer with the photoresist layer is then carefully placed under the mask and held against it. The technique is called contact lithography. Then the mask-wafer assembly is illuminated by a 194-W UV light source for $30 \mathrm{~s}$. The UV light transmits through to the transparent areas of the mask and chemically softens the photoresist layer. However, the photoresist layer beneath the opaque areas remains chemically hard. A postexposure bake at $90^{\circ} \mathrm{C}$ for $60 \mathrm{~s}$ is used to mechanically harden the photoresist.

7. A developer solution AZ-300-MIF is used to dissolve the chemically soft parts of the photoresist layer. The postbaked UV treated wafer is dipped in the developer solution and shaken for a minute (total) in two 30-s intervals. The UV-softened areas of the photoresist layer get dissolved and holes are created on the photoresist layer. The wafer is then washed with distilled water and dried, leading to the cleaning of the developer solution.

\subsection{Etching the Wafer: The Bosch Process}

Deep reactive ion etching (DRIE), or the Bosch process, ${ }^{29}$ of silicon, enables versatile and uniform microfabrication of high-aspect-ratio structures using the high etching rate of fluorine rich plasmas and the deposition of inhibiting films to obtain anisotropic profiles. An etching cycle, flowing only $\mathrm{SF}_{6}$, is alternated with a sidewall-passivating cycle using only $\mathrm{C}_{4} \mathrm{~F}_{8}$ (Fig. 8).

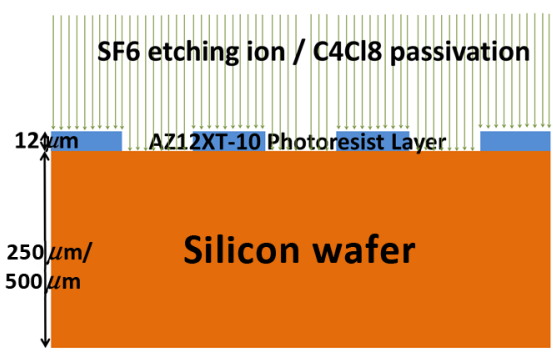

(a)

Through hole
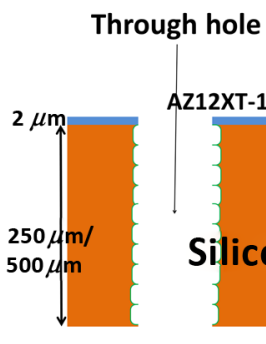
AZ12XT-10 Photoresist layer

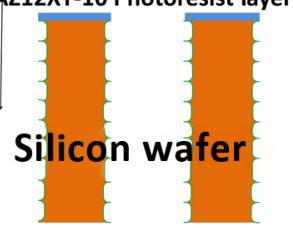

SF6 etching ion / C4Cl8 passivation

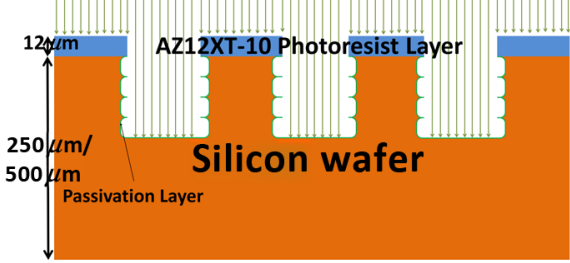

Through hole

(b)

(c)

Fig. 8 Silicon wafer time-multiplexed deep etching process schematic. $\mathrm{SF}_{6}$ is used as the etching reagent while $\mathrm{C}_{4} \mathrm{Cl}_{8}$ used as the passivating element. (a) Etching level at the start of etching/ passivation cycle, (b) etching level at the middle of Bosch process, and (c) fully etched silicon wafer. 
Table 1 Bosch DRIE process parameter values for fiber holders.

\begin{tabular}{llcl}
\hline \hline Quantity & Unit & Value & Comment \\
\hline Pressure & mTorr & 20 & A variation of 17-21 is seen \\
Coil power & Watt & 640 & \\
Electrode power & Watt & 12 & Slightly higher value is also acceptable \\
Bias & Volt & 80 & \\
Passivation time & Second & 6 & Can be changed to 7 s for thicker wafer \\
Etching time & Second & 10 & \\
Passivator flow rate & sccm & 96 & \\
Etchant flow rate & sccm & 101 & This is also accompanied by oxygen \\
Total process time & Minute & 150 & $\begin{array}{l}\text { Total time may vary depending on } \\
\text { the thickness of photoresist coating }\end{array}$ \\
\hline \hline
\end{tabular}

The $\mathrm{C}_{4} \mathrm{~F}_{8}$ deposits a Teflon-like film ${ }^{30}$ on the sidewalls to inhibit their etching during the subsequent $\mathrm{SF}_{6}$ cycle. This passivating film is preferentially removed from the bottom of the trenches due to ion bombardment. Because of the alternating between etching and passivating cycles, DRIE is also-perhaps more aptly—referred to as time-multiplexed deep etching. The alternating etching and passivating cycles lead to scallops on the sidewalls of etched structures. The peak-to-valley height of these scallops can be controlled by operating conditions.

Experimental studies ${ }^{30,31}$ suggest that the achievable fillet radii, surface quality, and etch rate are functions of the etch conditions, namely the flow rate of $\mathrm{C}_{4} \mathrm{~F}_{8}$ and $\mathrm{SF}_{6}$, electrode power, chamber pressure, and etching cycle duration. Developing through-holes with vertical walls requires a careful combination of these parameters. The parameters we adopted are given in Table 1 are given by WCAM through a preinstalled recipe. However, we tweaked passivation and etching time, electrode power in the given prescription to suit our requirements.
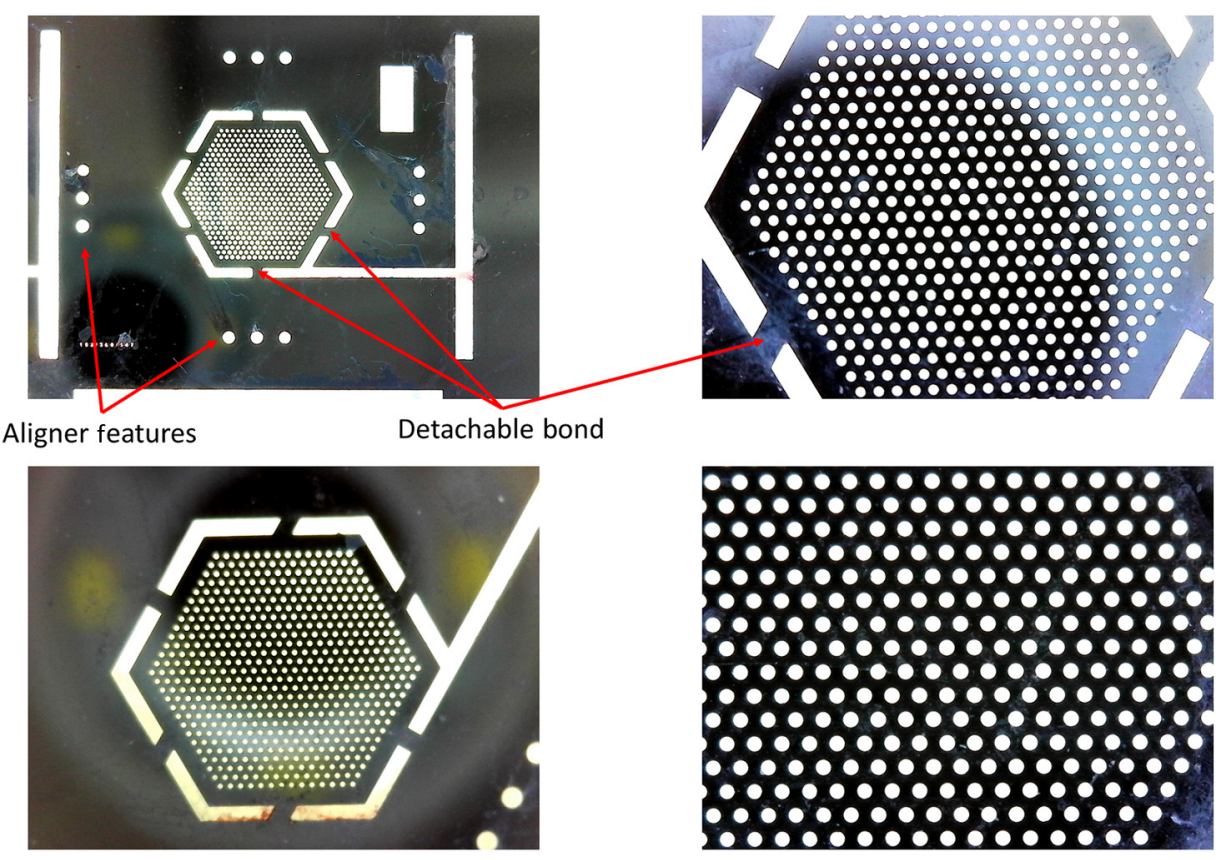

Fig. 9 Back illuminated image of a typical fiber array (250- $\mu \mathrm{m}$ thickness) manufactured at WCAM viewed at different magnifications. 


\subsection{Performance of Photolithography}

Hole positions and their diameters of the fiber holders developed through photolithography have been measured from microscope images using a python script. The script takes a back-illuminated image of a hole array as input and fits an ellipse to each bright spot corresponding to a hole, as shown in Fig. 9. The fitted minor axis is defined as the hole diameter, whereas the centroid of the ellipse is defined as the center of the hole. The measurement precision is driven by the signalto-noise ratio (SNR) as well as the pixel dimension and array size of the imaging camera. We have kept the SNR high in order to measure the position with arbitrary precision. The imaging area is quite large (an 8-mm-diameter area), whereas the USB microscope could only resolve up to $6.5 \mu \mathrm{m} /$ pixel. We rotated the array multiple times and repeated the measurement procedure. The measurement error of diameter and position across different measurements is found to be $\pm 0.8-\mu \mathrm{m}$ RMS.

We have etched two 500- $\mu \mathrm{m}$ and two $250-\mu \mathrm{m}$-thick-silicon wafers, each containing six hexagonal masks of 547 holes. The thicker wafers failed to yield consistent hole diameters and position as can be seen in Figs. 10 and 11. This can be primarily attributed to the variation between silicon and photoresist etch rate. For the given etch and passivation time (10 and $6 \mathrm{~s}$, respectively) to achieve vertical walls, the used pressure level $(90 \mathrm{mT})$ produced silicon to photoresist etch ratio of $\sim 21: 1$. The etch ratio is defined as the ratio of silicon and photoresist thickness etched over the same time scale. This implies we would require a photoresist thickness of $\sim 24 \mu \mathrm{m}$ to etch the 500- $\mu \mathrm{m}$ wafer. Such photoresist thickness is not achievable with the available photoresist material. Other photoresist material that can provide the required thickness can be used with metal only and not silicon. However, metal substrates cannot take advantage

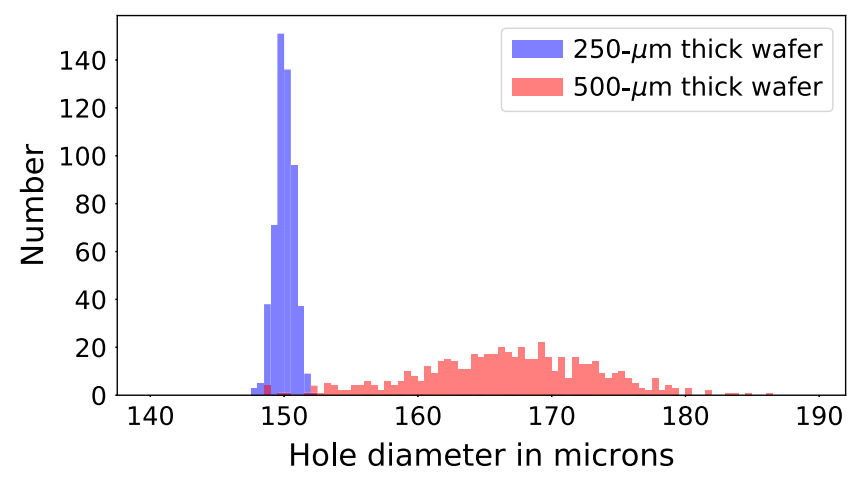

Fig. 10. Error distribution in the hole diameter for a typical mask from a $250-\mu \mathrm{m}$ and a $500-\mu \mathrm{m}$ wafer after performing photolithography at WCAM.

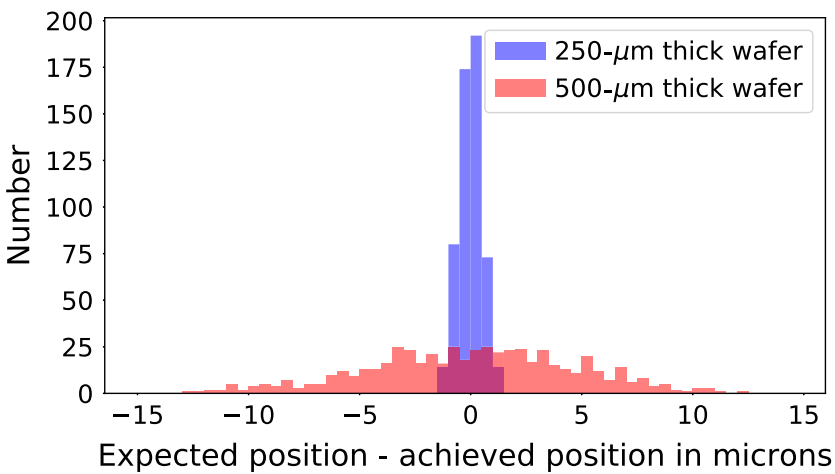

Fig. 11 Error distribution in hole relative position for a typical mask from a $250-\mu \mathrm{m}$ and a $500-\mu \mathrm{m}$ wafer after performing photolithography at WCAM. 
of the Bosch process (high-accuracy hole diameter and position). Although we could change the pressure to achieve a higher etch ratio, it would be very difficult to achieve etching-time and hole-diameter uniformity. Increasing the etch ratio would mean we would not have sufficient control on the horizontal etching of walls which may lead to inaccurate hole diameters. We could also modulate the electrode power and coil power to reduce the photoresist etch rate and, in turn, increase the etch ratio. The required coil and electrode power ( $<400$ and $<5 \mathrm{~W}$, respectively) would decrease the silicon etch rate thus reducing the etch ratio. This was found during the etching of 500- $\mu \mathrm{m}$ wafer: The photoresist was etched out within $2.5 \mathrm{~h}$, whereas the silicon wafer etching did not get through the entire wafer thickness. Once the photoresist was gone, the plasma would start etching the bare silicon. As a result, the hole diameter accuracy becomes unacceptable.

Etching of 250- $\mu \mathrm{m}$ produced holders with 1- and 0.5- $\mu \mathrm{m}$ RMS accuracy in hole diameter and relative position, respectively, as shown in Figs. 10 and 11. The recipe is repeatable and has a yield of $100 \%$. Since we need to stack 12 wafers to attain a thickness of $3 \mathrm{~mm}$, additional features have been etched to align and hold the wafers to make the fiber insertion easier, as shown in Fig. 7. We find that the mean and RMS error of diameters and positions do not vary across different masks on a wafer, as shown in Fig. 12. To demonstrate the suitability of these masks to achieve our thickness requirement, we have stacked all the wafers and found that they align together to form a clean hole for each fiber position, as shown in Fig. 13.

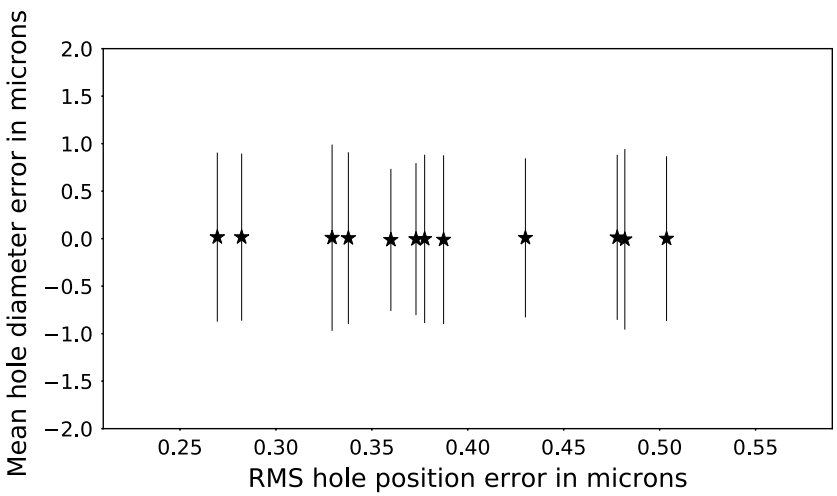

Fig. 12 Mean and peak to peak error in hole diameter against RMS relative position error for each of 12 masks from the two $250 \mu \mathrm{m}$ wafers processed via photolithography at WCAM.

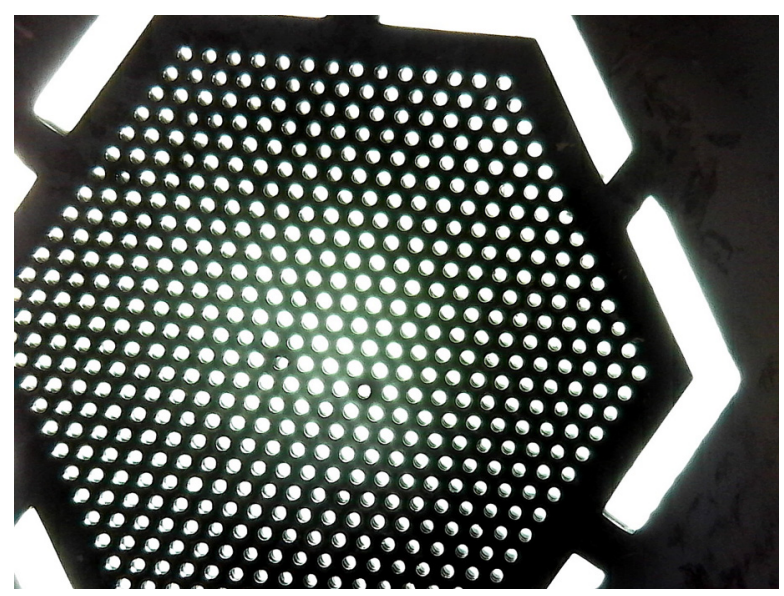

Fig. 13 Back-illuminated image of 12 stacked masks, each with $250-\mu \mathrm{m}$ thickness, to produce a fiber-holder with a total thickness of $3 \mathrm{~mm}$. 


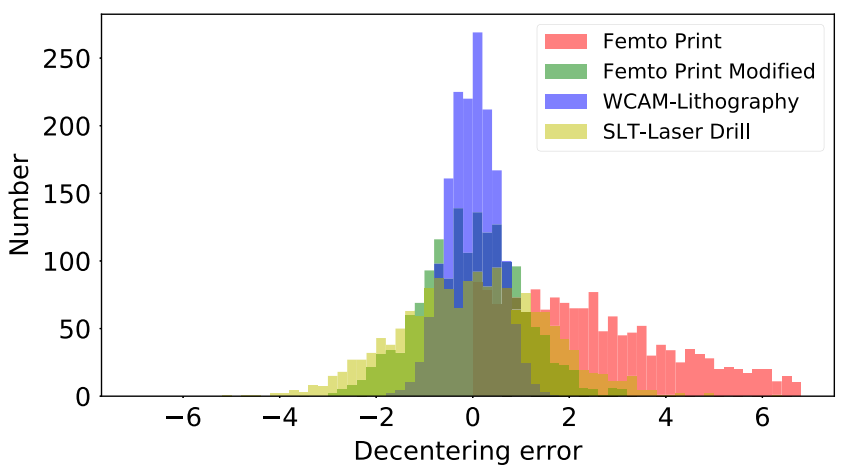

Fig. 14 Decenter distributions of the three fiber holder fabrication methods considered in this study (SLT, WCAM, and FP).

\section{Results}

\subsection{Optimized Core Filling}

We have generated three decenter distributions for a 2000-fiber array assuming Gaussian errors in positions with RMS values as described in Sec. 3.1 for the three methods shown in Fig. 14. The number of spaxels is large enough to avoid small-sample bias in a Gaussian distribution of random numbers. For each distribution, the IFU merit function $M_{i}$ is computed from Eq. (3) for a range of fiber-core input filling $\left(0.75<A_{i} / A_{c}<1.15\right.$, where $A_{c}$ is the fiber core area). Given our instrument configuration defined in Sec. 2.2, we adopt a fixed $\Omega_{i}$ corresponding to an input beam speed of $f / 4.2$, and a fixed $A_{o} \Omega_{o}$ for a fully illuminated fiber exit core with $A_{o}=A_{c}$ and $f / 4.2$ output $f$-ratio (i.e., ideal fiber without FRD). As shown in Fig. 15, the merit function increases with $A_{i}$ until $A_{i} / A_{c}$ approaches $97 \%$, beyond which the EE term in the merit function begins to dominate as light is lost outside of the over-filled fiber core.

Different technologies differ in providing positioning precision. For example, FP initially did not provide as good a solution as SLT or WCAM, but their improved precision is on par with the other techniques. A filling of $\sim 97 \%$ to $98 \%$ of the fiber core is the optimal choice, delivering a peak in the merit function that is only $6 \%$ lower than the ideal value (of unity). This optimum core filling fraction is independent of the number of spaxels (as the merit function is an average); fiber core size (as the fill fraction is a ratio of areas); and input or output focal ratio. The amplitude of the merit function does depend on the ratio of the input-to-output $f$-ratios; here we have assumed the case without FRD such that the input and output $f$-ratios are equal. We have no

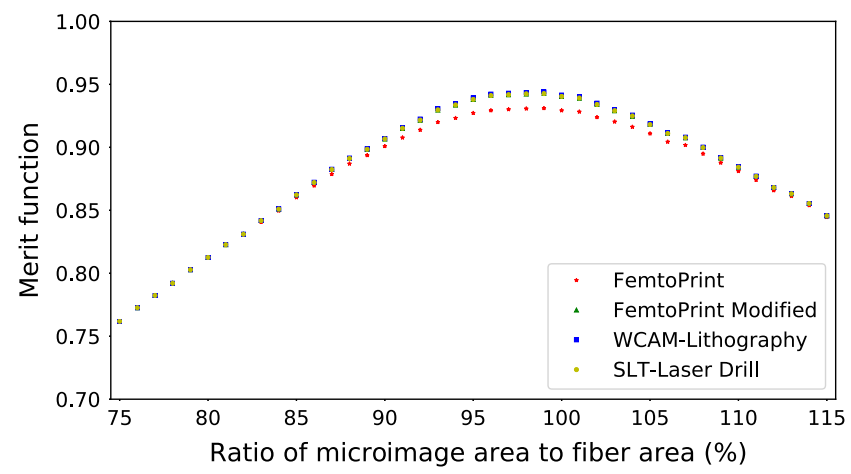

Fig. 15 Optimization of the fiber-core filling fraction (given as \%) based on our merit function for different hole positioning and diameter accuracies of three different fiber-holder fabrication methods (SLT, WCAM, and FP). The merit function assumes an ideal fiber with no FRD, but this assumption only impacts the merit function amplitude not the location of the maximum with respect to the filling fraction. A core filling fraction of $97 \%$ to $98 \%$ provides the least loss in terms of input photons and observing efficiency. This result is independent of the fiber core size as well as the input and output focal ratios. 


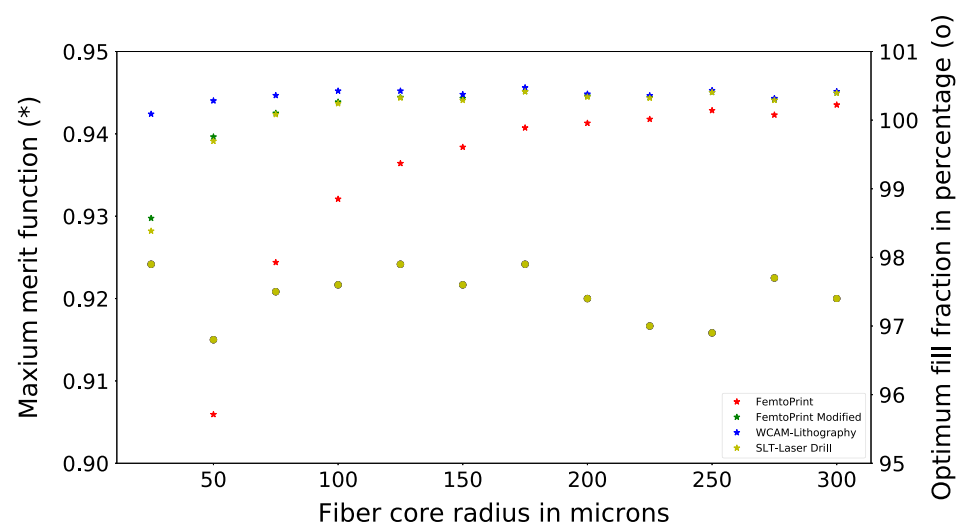

Fig. 16 Variation of maximum achievable merit function and optimum fill fraction against various fiber core dimensions for different techniques of fiber holder development in an ideal fiber having no FRD. The optimum core fill fraction is same for all the techniques given a fixed core size and varies around $97 \%$ to $98 \%$ across all core sizes.

evidence that FRD depends on the core filling fraction. We assume a contiguous distribution of microlenses for the best possible application of the microlens-coupled fiber IFU technique.

It is also interesting to note that although SLT/Modified FP hole positioning accuracy is lower than WCAM-lithography, we are not losing much in terms of the merit function maximum value. This primarily stems from the fact that the difference between input spot and fiber core diameters remains comparable to the fiber decenter distribution for SLT and modified FP methods; by increasing the fiber positioning we would gain neither in throughput nor in observing efficiency for a core diameter of $100 \mu \mathrm{m}$. However, changing the core size would change this scenario as shown in Fig. 16 which describes the variation of maximum achievable merit function and optimum fill fraction from different techniques against core diameter. The optimum fill fraction for a given fiber core radius remains the same across all techniques and also distributed $\sim 97 \%$ to $98 \%$ for different core sizes. For the smaller fibers ( $\leq 100-\mu$ m radius), the difference between core and microimage diameter would be equivalent to decenter for WCAM but much smaller for modified FP and SLT. As the core diameter grows, for the same fill fraction, the difference gets higher and eventually becomes equivalent to the decenter distribution of SLT and modified FP and at this point, they perform very similar to WCAM.

\subsection{Fiber Holder Thickness Requirements}

In Sec. 2.3, we have described the thickness required for the fiber holder that would hold the fiber array for the SMI instrument for the SALT. Given the three available techniques, here we present

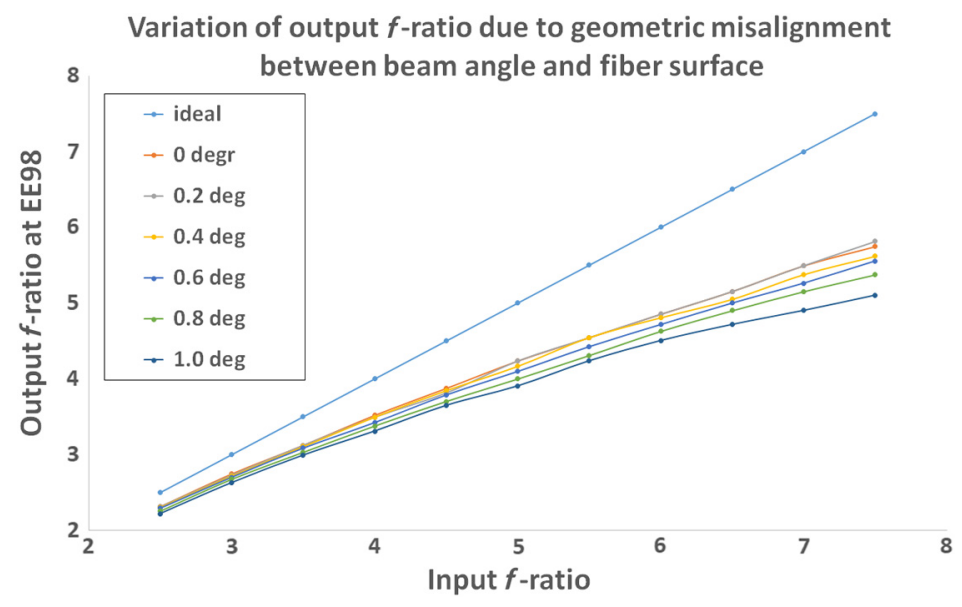

Fig. 17 Effect of misalignment between fiber face and optical beam (termed as tilt) on the output focal ratio without incorporating the optically introduced FRD. 


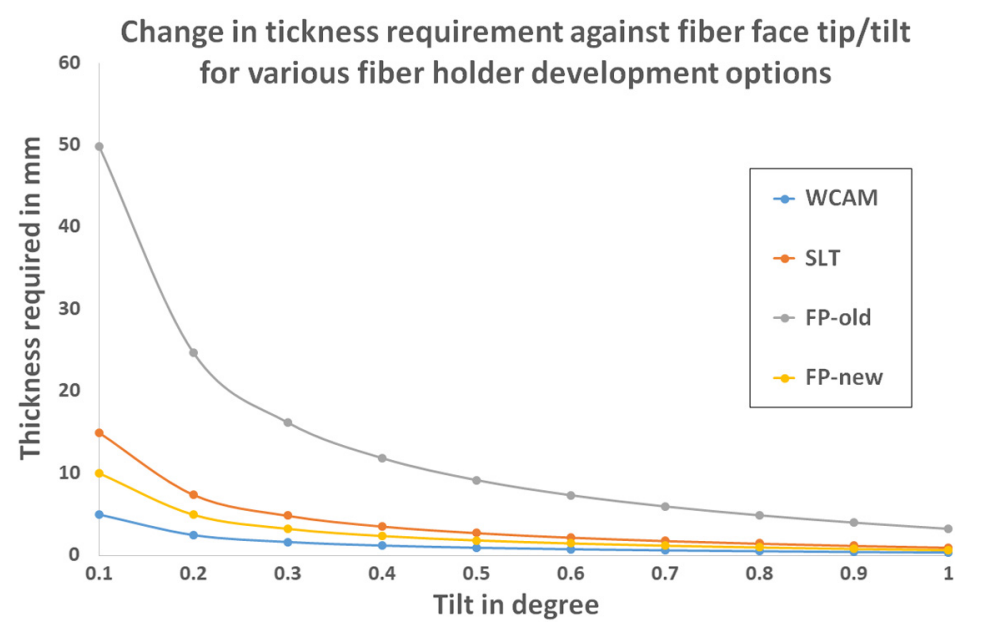

Fig. 18 Variation in fiber holder thickness requirement for different tilt angles and different holder development techniques.

a generic treatment toward defining the required thickness of a fiber holder. Figure 17 shows the variation of output focal ratio due to misalignment between the input beam and the fiber face. This tip/tilt can be converted to a thickness requirement based on the fiber hole diameter accuracy of different fiber holder development techniques as provided in Sec. 3.1. The change in the thickness required to achieve a range of tilt angle has been shown in Fig. 18. At a given tilt tolerance, the thickness requirement decreases with increasing hole diameter accuracy. For a tilt of $0.2 \mathrm{deg}$, WCAM needs the smallest thickness of $2.5 \mathrm{~mm}$. At a faster input focal ratio $(<f / 3.5)$, the optomechanical design of the IFU can accept tilts up to $1 \mathrm{deg}$, in which all of the holder development techniques provide equivalent thickness requirement $(<1 \mathrm{~mm})$.

\section{Summary}

Developing multispaxel microlens-fiber coupled IFUs requires careful alignment within $\sim 1 \%$ of the fiber diameter. Over or underfilling the fiber core with the microimage can lead to severe light-loss. We find $97 \%$ to $98 \%$ is the ideal filling fraction of the fiber core with the microimage/ micropupil for a fiber-positioning accuracy of $<1-\mu \mathrm{m}$ RMS. In addition to positioning accuracy, the fiber holder must minimize the tilt between fiber and the microlens to minimize gFRD. An acceptable tilt of $0.1 \mathrm{deg}$ will ensure $f / 4$ output for an input beam of $f / 4.2$ at $98 \%$ EE. Several methods of fiber holder development have been pursued and the effect of their drilling uncertainties on throughput has been analyzed. It is found that FP, SLT, and WCAM can be used with equal efficiency. The process of photolithography (WCAM) has been deployed to etch 250-and 500- $\mu$ m-thick-silicon wafers. The recipe was successful for the thinner wafer, fulfilling the desired hole position and diameter accuracy of $\sim 0.5$ - and 1- $\mu \mathrm{m}$ RMS, respectively, with a yield of $100 \%$.

The three possible techniques can be used to achieve similar results but at different costs. FP can produce holders of 5-mm thickness and thus stacking errors are not present but we found that this is the costliest among the three techniques. Fiber holders manufactured at SLT may or may not require stacking depending on the fiber size as the hole diameter to drill thickness is fixed (1:10); individual SLT plates are lower cost than FP. Holders fabricated at WCAM must be stacked as the individual wafer thickness can only be $250 \mu \mathrm{m}$. The stacking error may be mitigated with aligning features and the process cost is significantly cheaper than the other two. However, for WCAM, labor costs bring the total cost much closer to the other techniques for a small production set. An instrument requiring several IFUs (similar to SMIFU specification) may find WCAM costs to be competitive with the other techniques since the labor does not increase significantly as the process of photolithography can be made parallel (up to three wafers with the current facility) as well as multiple wafers can be etched simultaneously. It is difficult to generalize the relative costs of the different method since they depend on the specific application, 
but we conclude below with the relative costs for our application and indicate how these relative costs change depending fiber and array size as well as tilt requirement.

For our nominal case requiring $<0.1 \mathrm{deg}$ fiber tilt for $200 \mu \mathrm{m}$ core fibers, leading to 3-mm holder thickness, WCAM lithography is about $10 \%$ cheaper than SLT and a factor of 4 less than FP. Keeping the number of fibers and the tilt requirement fixed, for larger fiber diameter $(>500 \mu \mathrm{m})$ SLT becomes the most cost-effective approach, followed by WCAM and then FP. This change in relative cost comes about because SLT's technique is limited by aspect ratio, and this becomes salient for large fiber sizes. For stricter tilt requirement, this trend continues. On the other hand for large fiber diameters $(>500 \mu \mathrm{m})$ and loose tilt criteria $(>0.1 \mathrm{deg}) \mathrm{FP}$ becomes cheaper followed by WCAM and SLT. At small fiber diameters $(<100 \mu \mathrm{m})$ and tight tilt criteria $(<0.025 \mathrm{deg})$ FP and SLT are comparable but remain factors of a few more expensive than WCAM.

Overall, we were impressed with FP's capabilities and performance, SLT's performance and low cost, and WCAM's facilities for in-house custom-development. For instrument projects without in-house access to superb photolithorgraphic facilities such as WCAM, or without personnel trained to use such facilities, these commercial vendors, or ones like them, should serve as essential for successful, higher-performance fiber-lenslet coupling.

\section{Acknowledgments}

This research was supported by funds from the University of Wisconsin-Madison Graduate School, National Science Foundation AST-1517006. The authors gratefully acknowledge the use of facilities and instrumentation at the UW-Madison Wisconsin Centers for Nanoscale Technology (wcnt.wisc.edu) partially supported by the National Science Foundation through the University of Wisconsin Materials Research Science and Engineering Center (DMR1720415). We also thank Frank Pfefferkorn for his input toward vendor choice and fabrication options.

\section{References}

1. S. C. Barden and R. A. Wade, "DensePak and spectral imaging with fiber optics," Astron. Soc. Pac. Conf. Ser. 3, 113-124 (1988).

2. A. Kelz et al., "PMAS: the potsdam multi-aperture spectrophotometer. II. The wide integral field unit PPAK," Publ. Astron. Soc. Pac. 118(839), 129-145 (2006).

3. N. Drory et al., "The MaNGA integral field unit fiber feed system for the Sloan $2.5 \mathrm{~m}$ telescope," Astron. J. 149, 77 (2015).

4. J. J. Bryant and J. Bland-Hawthorn, "Square-core bundles for astronomical imaging," Proc. SPIE 8446, 84466K (2012).

5. M. A. Bershady et al., "SparsePak: a formatted fiber field unit for the WIYN telescope bench spectrograph. I. Design, construction, and calibration," Publ. Astron. Soc. Pac. 116, 565590 (2004).

6. G. J. Hill et al., "Design, construction, and performance of VIRUS-P: the prototype of a highly replicated integral-field spectrograph for HET," Proc. SPIE 7014, 701470 (2008).

7. M. H. Fabricius et al., "VIRUS-W: an integral field unit spectrograph dedicated to the study of spiral galaxy bulges," Proc. SPIE 7014, 701473 (2008).

8. D. R. Law et al., "Observing strategy for the SDSS-IV/MaNGA IFU galaxy survey," Astron. J. 150, 19 (2015).

9. R. L. Davies et al., "GMOS: the GEMINI multiple object spectrographs," Proc. SPIE 2871, 1099-1106 (1997).

10. L. Pasquini et al., "FLAMES: a multi-object fiber facility for the VLT," Proc. SPIE 4008, 129-140 (2000).

11. O. LeFevre et al., "Commissioning and performances of the VLT-VIMOS," Proc. SPIE 4841, 1099-1106 (2003).

12. A. G. de Paz et al., "MEGARA, the new intermediate-resolution optical IFU and MOS for GTC: getting ready for the telescope," Proc. SPIE 9908, 99081K (2016). 
13. X.-T. Yan et al., "Design of the microlens arrays coupling with imaging fiber bundle," Optoelectron. Lett. 9, 169-172 (2013).

14. S. C. Ellis et al., "KOALA: a wide-field 1000 element integral-field unit for the AngloAustralian Telescope," Proc. SPIE 8446, 84460V (2012).

15. G. Avila, "FRD and scrambling properties of recent non-circular fibres," Proc. SPIE 8446, 84469L (2012).

16. J. F. P. Spronck et al., "Extreme Doppler precision with octagonal fiber scramblers," Proc. SPIE 8446, 84468T (2012).

17. S. Halverson et al., "An efficient, compact, and versatile fiber double scrambler for high precision radial velocity instruments," Astrophys. J. 806, 61 (2015).

18. M. P. Smith et al., "Pupil scrambling integral field unit (PSI) for the Robert Stobie spectrograph on SALT," Proc. SPIE 9912, 99125B (2016).

19. D. Ren and J. Allington-Smith, "On the application of integral field unit design theory for imaging spectroscopy,” Publ. Astron. Soc. Pac. 114(798), 866-878 (2002).

20. A. D. Eigenbrot, M. A. Bershady, and C. M. Wood, "The impact of surface-polish on the angular and wavelength dependence of fiber focal ratio degradation," Proc. SPIE 8446, 84465W (2012).

21. J. Allington-Smith et al., "End effects in optical fibres," Mon. Not. R. Astron. Soc. 436, 3492-3499 (2013).

22. E. Carrasco and I. R. Parry, "A method for determining the focal ratio degradation of optical fibres for astronomy," Mon. Not. R. Astron. Soc. 271, 1-12 (1994).

23. C. G. Wynne, "Telecentricity in fibre-fed spectrographs," Mon. Not. R. Astron. Soc.y 260, 307-316 (1993).

24. R. Yan et al., "SDSS-IV MaNGA IFS galaxy survey-survey design, execution, and initial data quality," Astron. J. 152, 197 (2016).

25. R. Yan et al., "SDSS-IV/MaNGA: spectrophotometric calibration technique," Astron. J. 151, 8 (2015).

26. A. Pérez-Calpena et al., "MEGARA: high-precision alignment system for gluing fibers and microlenses," Proc. SPIE 9912, 99125L (2016).

27. M. Bass et al., Handbook of Optics, Third Edition Volume II: Design, Fabrication and Testing, Sources and Detectors, Radiometry and Photometry, McGraw-Hill, Inc., New York (2009).

28. S. Chattopadhyay et al., "A new photolithography based technique to mass produce microlens + fibre based integral field units (IFUs) for 2D spectroscopy," Proc. SPIE 10706, 107062D (2018).

29. E. H. Klaassen et al., "Silicon fusion bonding and deep reactive ion etching: a new technology for microstructures," Sens. Actuators A: Phys. 52(1), 132-139 (1996).

30. A. A. Ayón et al., "Deep reactive ion etching of silicon," MRS Proc. 546, 51 (1998).

31. A. A. Ayón et al., "Characterization of a time multiplexed inductively coupled plasma etcher," J. Electrochem. Soc. 146(1), 339-349 (1999).

Sabyasachi Chattopadhyay is currently a postdoctoral research fellow at the South African Astronomical Observatory working on the slit mask integral field unit for the SALT. He has worked at the University of Wisconsin-Madison after receiving his $\mathrm{PhD}$ in astronomy from the Inter University Center for Astronomy and Astrophysics, Pune, for developing DOTIFS. His field of interest is fiber spectroscopic instrumentation and optical detector control.

Matthew A. Bershady is a professor in the Department of Astronomy and Ciriacks Faculty Fellow of the College of Letters and Science at the University of Wisconsin-Madison, where he currently serves as a project scientist for the Sloan Digital Sky Survey-IV. He is also a South African Research Chair at the South African Astronomical Observatory and in the Department of Astronomy at the University of Cape Town.

Marsha J. Wolf received her BS degree in electrical engineering from Kettering University in 1986, her MS degree in electro-optics from the University of Dayton in 1991, and her PhD in astronomy from the University of Texas at Austin in 2005. She is a senior scientist at the 
University of Wisconsin-Madison. Her research interests include astronomical instrumentation and the co-evolution of galaxies and their AGN. She is a member of SPIE.

Michael P. Smith is a mechanical engineer who has been working on astronomy projects for 18 years at both the University of Wisconsin and University of Texas. He has played major roles in the development of instruments for the Southern African Large Telescope, Hobby Eberly Telescope, the WIYN Telescope, and the Sloan Digital Sky Survey. Projects have included the development of spectrographs (visible and near-infrared), detector systems, fiber integral field units, and telescope subsystems.

Andrew Hauser: Biography is not available. 